\section{Targeting cardiolipin in pneumonia}

\section{By Tim Fulmer, Senior Writer}

U.S. researchers have developed a new way for treating pneumonia resistant to standard first-line therapy with broad-spectrum antibiotics. Instead of attacking the bacteria, the researchers designed a peptide that binds cardiolipin, a host-generated lipid involved in lung tissue inflammation and injury. ${ }^{1}$

Cardiolipin is found almost exclusively in the membranes of mitochondria, in which it helps maintain membrane architecture and provides stability for enzymes involved in energy metabolism. ${ }^{2,3}$ Although the lipid exists at low concentrations in healthy lung fluid, previous studies have shown that markedly higher levels of cardiolipin occur in animal models of lung injury and inflammation., ${ }^{4,5}$

Those findings suggest that the mechanism for regulating cardiolipin is disrupted as a result of disease, leading to excessive levels of the lipid in lung fluid that contribute to tissue injury. The open questions have been where the mechanism breaks down and whether it could be restored to lower cardiolipin levels and improve lung function.

"Moving forward, we hope
to eventually develop a
way of directly delivering
these cardiolipin-binding
peptides to specific regions
of the patient's lung using a
bronchoscope."
- Rama Mallampalli,
University of Pittsburgh

To answer those questions, a research team led by Rama Mallampalli, professor of medicine at the University of Pittsburgh and chief of the Pulmonary Division at the VA Pittsburgh Healthcare System, connected the dots between cardiolipin and a membrane lipid pump called ATPase aminophospholipid transporter class I type 8B member 1 (ATP8B1; FIC1).

Previous work by other groups has shown that loss-of-function mutations in ATP8B1 increase the risk of developing pneumonia. ${ }^{6}$

Given that ATP8B1 plays an important role in maintaining lipid levels in cell membranes, Mallampalli's team hypothesized that the lipid pump might be a component of cardiolipin's regulatory mechanism. If so, restoring ATP8B1 function could decrease cardiolipin levels and treat pneumonia.

The researchers first confirmed that pneumonia patients had significantly higher levels of cardiolipin in their tracheal aspirates than those in aspirates from controls with nonpulmonary illnesses $(p=0.0007)$.

In mice infected with strains of Haemophilus influenzae or pneumonia-causing Escherichia coli, the bronchoalveolar lavage fluid had significantly higher levels of cardiolipin than fluid from uninfected controls $(p<0.01)$.

The team then found that impairing the function of Atp8b1 in the mice significantly increased levels of bronchoalveolar lavage cardiolipin compared with those in wild-type controls $(p<0.01)$ while also impairing lung mechanics $(p<0.05)$.

In contrast, forced overexpression of Atp $8 b 1$ in the diseased mice led to lower levels of cardiolipin in the lung and reversed impairments in lung mechanics.

Finally, the researchers looked at whether delivering the cardiolipinbinding domain of ATP8B1 would be sufficient to bind and sequester excess cardiolipin and treat pneumonia.

In mice infected with pneumonia-causing E. coli, intratracheal delivery of a peptide expressing Atp8bl's cardiolipin-binding domain improved three different parameters of lung mechanics and also significantly increased survival ( $p=0.001)$ compared with delivery of vehicle control.

Results were published in Nature Medicine.

The authors concluded: "These results may eventually serve as a springboard to generate drug therapies to sequester or enhance clearance of injurious cardiolipin in pneumonia."

Corresponding author Mallampalli told SciBX that the team now wants to improve on the cardiolipin-binding peptide by developing analogs with better specificity for cardiolipin.

"Moving forward, we hope to eventually develop a way of directly delivering these cardiolipin-binding peptides to specific regions of the patient's lung using a bronchoscope," he said. "The cardiolipin-binding peptide strategy could be of particular use in situations where patients show resistance to the broad-spectrum antibiotics that are the standard, first-line treatment of pneumonia."

Mallampalli did not disclose the patent or licensing status of his findings.

Fulmer, T. SciBX 3(39); doi:10.1038/scibx.2010.1170

Published online Oct. 7, 2010

\section{REFERENCES}

1. Ray, N.B. et al. Nat. Med.; published online Sept. 19, 2010; doi:10.1038/nm.2213

Contact: Rama K. Mallampalli, University of Pittsburgh, Pittsburgh, Pa. e-mail: mallampallirk@upmc.edu

2. Chicco, A.J. \& Sparagna, G.C. Am. J. Physiol. Cell Physiol. 292, C33-C44 (2007)

3. Houtkooper, R.H. \& Vaz, F.M. Cell Mol. Life Sci. 65, 2493-2506 (2008)

4. Liau, D.F. et al. J. Lipid Res. 25, 678-683 (1984)

5. Ksenzenko, S.M. et al. J. Appl. Physiol. 82, 2020-2027 (1997)

6. Pawlikowska, L. et al. J. Hepatol. 53, 170-180 (2010)

COMPANIES AND INSTITUTIONS MENTIONED University of Pittsburgh, Pittsburgh, Pa. VA Pittsburgh Healthcare System, Pittsburgh, $\mathrm{Pa}$. 